\title{
COMPARING THE IDENTIFICATION OF RECOMMENDATIONS BY DIFFERENT ACCIDENT INVESTIGATORS USING A COMMON METHODOLOGY
}

\author{
Chris W. Johnson, H.A. Oltedal*, C.M. Holloway ${ }^{\dagger}$ \\ School of Computing Science, University of Glasgow, Scotland. G12 8RZ, Scotland. \\ *Stord/Haugesund University College, Haugesund, Norway. \\ ${ }^{\dagger}$ NASA Langley Research Center ,100 NASA Road / Mail Stop 130, Hampton VA 23681-2199 USA.
}

Keywords: Accident Investigation, SOL, Root Cause Analysis.

\begin{abstract}
Accident reports play a key role in the safety of complex systems. These reports present the recommendations that are intended to help avoid any recurrence of past failures. However, the value of these findings depends upon the causal analysis that helps to identify the reasons why an accident occurred. Various techniques have been developed to help investigators distinguish root causes from contributory factors and contextual information. This paper presents the results from a study into the individual differences that can arise when a group of investigators independently apply the same technique to identify the causes of an accident. This work is important if we are to increase the consistency and coherence of investigations following major accidents.
\end{abstract}

\section{Introduction}

Many different analytical techniques have been developed to support the identification of causal factors during accident investigations. Many of the proponents of these tools have argued that by using an agreed methodology, it is possible to increase the consistency of investigations [3]. In other words, similar causes will be identified for similar incidents. Accident analysis methods can also help to reduce individual bias; by encouraging investigators to follow and document common processes during an investigation [5]. A small number of previous studies have been conducted to determine the veracity of these arguments, for instance as part of the Bielefeld Workshop series [1]

This paper builds on previous work by presenting the results of an independent study involving three experienced analysts using common source materials, each applying the Safety through Organizational Learning (SOL) approach [7]. The focus of the analysis was a collision into mountainous terrain that occurred in Alaska during 2010. Many other analysis techniques might have been used, including Why-Because Analysis, Systems-Theoretic Accident Model and Processes (STAMP), or Events and Causal Factors charting [3]. SOL was chosen because it was intended to support the analysis of organisational and managerial factors in accident investigations.

This paper focuses on the differences among individual analysts rather than the choice of particular techniques. It is concluded that the application of common analysis tools does not guarantee agreement over potential recommendations. In contrast to previous research that has stressed the importance of inter-analyst agreement; we would argue that such differences encourage teams of investigators to consider a range of interventions prior to the publication of an accident or incident report.

\section{Summary of the Accident Case Study}

The decision was taken to base our work on an existing accident report, rather than attempt to conduct the research using a 'live' investigation. This was justified for pragmatic reasons; it can be difficult to ensure access to an on-going investigation. There were ethical reasons: ensuring that the findings of our analysis do not bias the recommendations that are essential to avoid the repetition of previous fatal accidents. This paper focuses on the loss of a single-engine, turbine-powered, amphibious float-equipped de Havilland DHC-3T. The pilot and four passengers received fatal injuries. Four other passengers received serious injuries. The flight took off from a private lodge on the shore of Lake Nerka at 14:27 towards a remote sport fishing camp about 52 $\mathrm{nm}$ southeast on the Nushagak River. The accident pilot was familiar with the route. His aircraft was equipped with two global positioning system (GPS) units with moving map and terrain display capabilities and a radar altimeter with visual annunciator and aural tone capabilities. The NTSB found that in spite of these navigational systems, the aircraft diverted from the expected course during the final minutes of the flight. It headed towards mountainous terrain and eventually crashed.

The investigation examined a range of human factors concerns that might have explained the pilot's deviation from the expected heading. These included fatigue as well as 'major life events'; including his retirement and the sudden death of a family member. The NTSB found that he had suffered an intracerebral hemorrhage four years before the 
accident. It was concluded that the probable cause was the pilot's temporary unresponsiveness 'for reasons that could not be established from the available information'. A number of recommendations were made in the final report [6]. In particular, they focussed on inadequate regulatory guidance for the medical certification of aircrew.

\section{Introduction to SOL}

Safety through Organizational Learning (SOL) is a general approach to analysing adverse events [2]. It has been widely used across many industries in several different countries and is one of a number of analytical tools and techniques that can be used to assist accident investigations $[1,3]$. The popularity and longevity of the approach make it an appropriate starting point for the identification of individual differences in the application of common approaches to mishap investigation. A further justification is that SOL emerged from several different strands of research within the area of 'sociotechnical systems analysis'. This is important because managerial and organisational factors are often the most complex to investigate and at the same time are amongst the most significant causes of incidents and accidents. SOL analysis considers the role of technology, individuals, teams, organisations and the environment. By considering this range of potential causes, analysts are encouraged to look beyond individual 'root cause'. The identification of the five common sub-systems arguably increases consistency between investigators - they must consider the same five areas of concern. The SOL analysis technique is based around a number of different stages:

1. Identify the events that occurred during the accident;

2. Organise these events into Event Building Blocks, these record the time, location, actor(s) involved and the action(s) that contributed to an incident or accident;

3. Arrange the Event Building Blocks into a TimeActor Diagram, this provides a time-line of events leading to an accident;

4. Identify contributing factors for each Event Building Block in the Time-Actor Diagram.

The direct and indirect factors associated with the Event Building Blocks include:
A. Representation of Information;
B. Communication;
C. Working conditions;
D. Personal performance;
E. Violation;
F. Technical components.

The SOL technique also considers a range of indirect factors including control and supervision; group influences; rules, procedures, and documentation etc. The following sections summarise the results that were obtained for three different investigators each applying the SOL analytical tools to the collision into mountainous terrain summarised in the opening sections of this paper. The intention is to illustrate the subjective differences that arise during the application of common analytical tools. Most of the attention is on the development of Time-Actor diagrams, which arguably lies at the heart of the SOL approach. The decision to focus on these artefacts is also justified by the difficulty of summarising the results without extending the descriptions across many pages of prose.

All three investigators have a similar background in terms of expertise and experience in the application of accident and incident investigation techniques, representing a cumulative total of more than 40 years of experience in the application of causal analysis tools. However, this expertise has been obtained across a range of investigations including software systems analysis, aviation applications and maritime accident analysis.

\section{Analyst A}

Figure 1 illustrates the results from an initial SOL analysis by the first analyst. It presents a Time-Actor diagram for the decision to issue the accident pilot with the medical certificate required to fly after his intracerebral hemorrhage (ICH) during March 2006. He made repeated attempts to regain certification. Under 14 CFR 67.109, "a transient loss of control of nervous system function(s) without satisfactory medical explanation of the cause" prevents pilots from gaining a medical certificate. 14 CFR 67.401 states that a medical certificate can be granted under special circumstances to a person who does not meet the regulatory provisions if they satisfy the Federal Air Surgeon or delegated authority that they can fulfil the associated duties without endangering public safety. The decision to allow the pilot to regain their medical certificate was also guided by the FAA's internal Aeromedical Certification Reference Manual; "special issuance consideration will be given to those who can demonstrate full recovery of motor, sensory, language, and intellectual function." The Alaska Regional Flight Surgeon's decision is shown as the bottom event in Figure 1.

Figure 2 extends the Time-actor diagram beyond the initial decision to re-issue the pilots medical certificate after he recovered from the $\mathrm{ICH}$. In particular, it denotes the possible impact of his recent bereavement and the observations by coworkers that this tragedy might have had upon the pilot. As mentioned above, subsequent stages of the SOL analysis build on these diagrams to identify a range of direct and indirect factors. Analyst A focused on communication issues and on regulatory concerns that led to the certification of the pilot, illustrated in Figure 1. In contrast, the following sections present interim results from the application of SOL by the remaining participants in the study. 


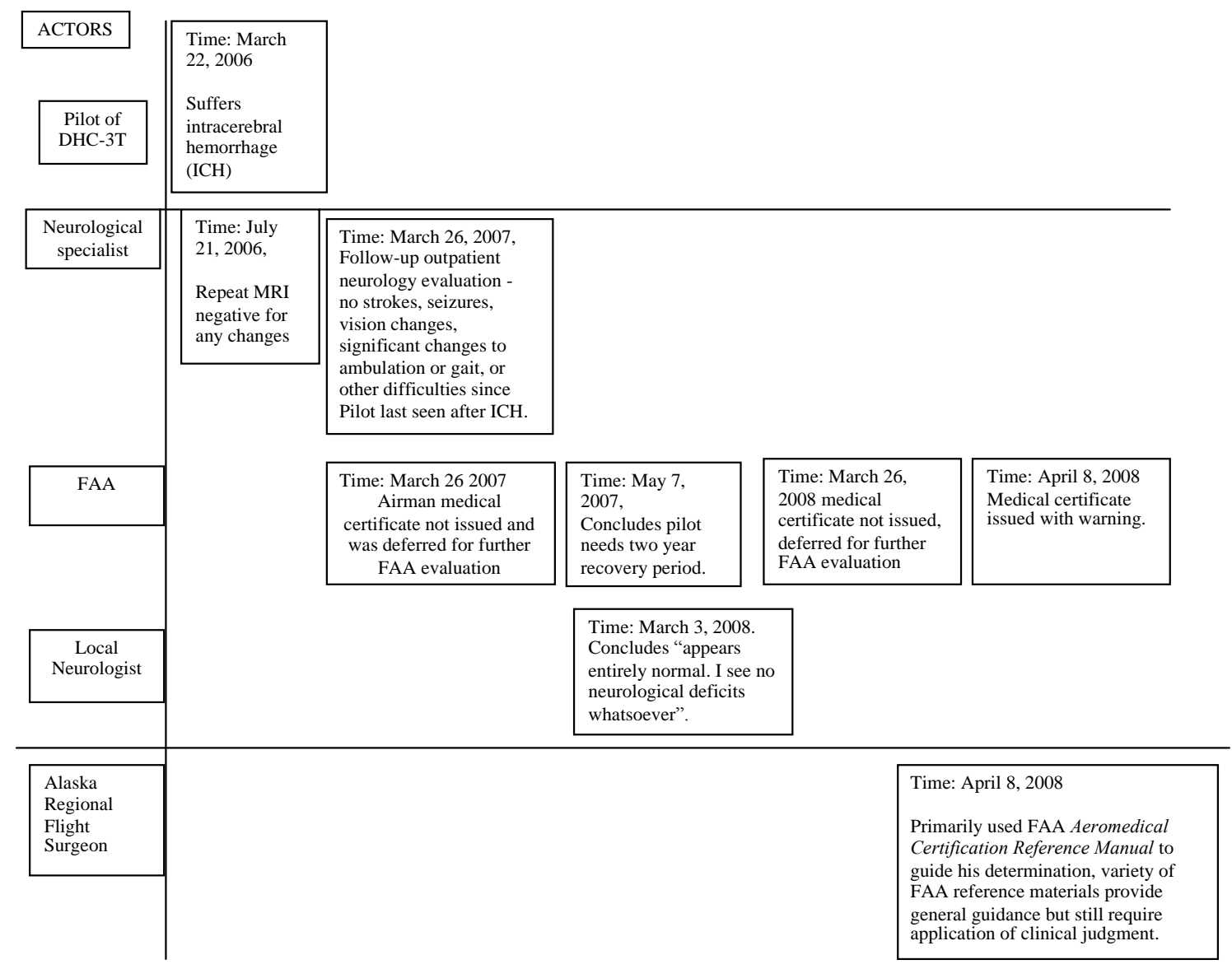

Figure 1: SOL Time-Actor Diagram for Analyst A, Focussing on FAA Certification of Pilot

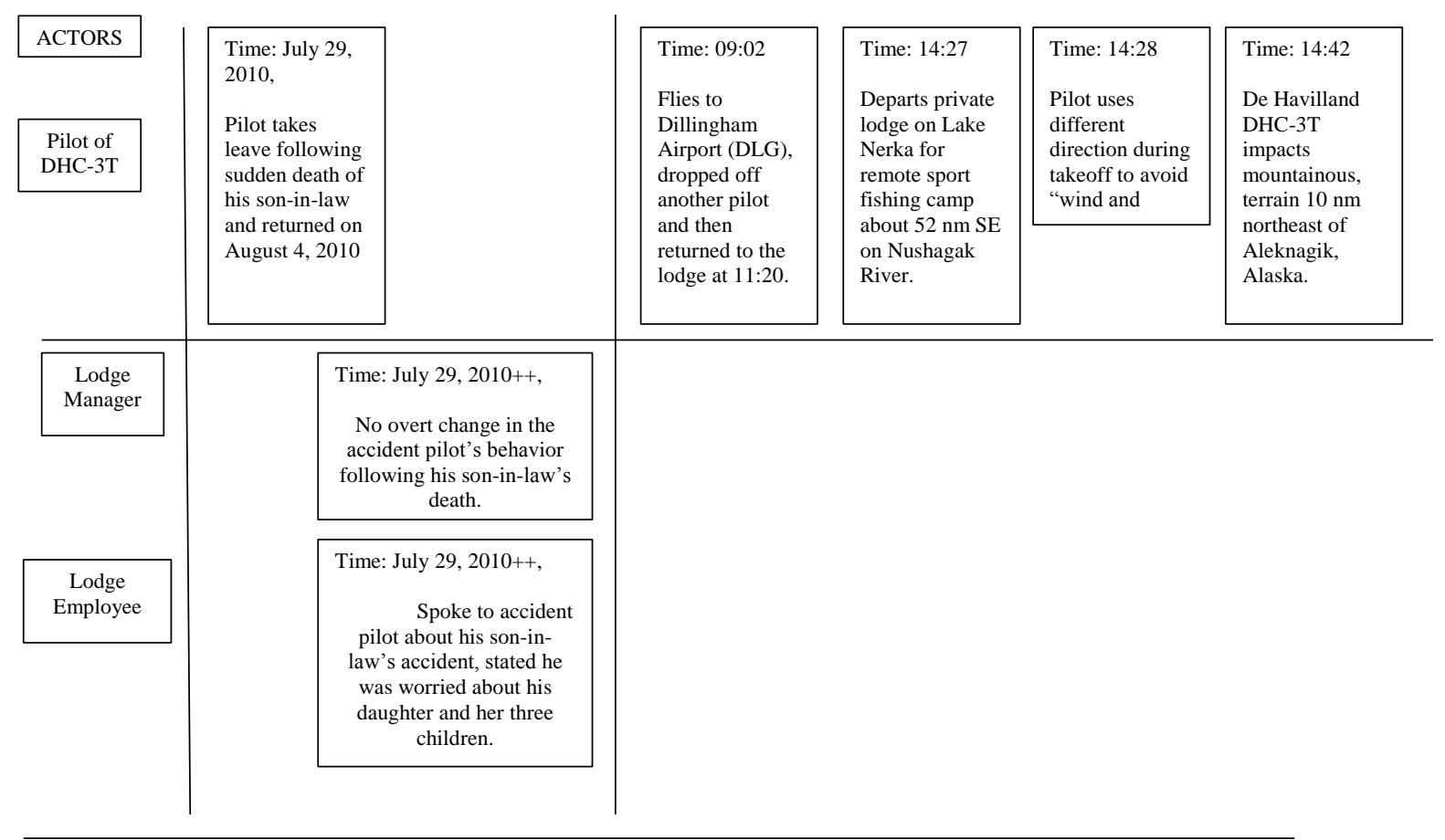

Figure 2: SOL Time-Actor Diagram for Analyst A, Immediate Events Prior to Collision With Mountainous Terrain 


\section{Analyst B}

Following the stages of the SOL analysis technique described in the introduction, analyst B began by identifying the events during the accident, i.e. what happened. An event is determined by a sequence of singular actions by different actors (maybe a person or a technical component) between a starting point and an end point. The starting point is defined as the first alarm or the first perceived deviation from a warranted course or action. According to the SOL guidelines the situational description should focus on observable facts only concerning what happened, without hypothesis about causes or why it happened [6]. After building the chain of events, Analyst B identified contributing factors. This aspect of SOL forms a strong contrast with other approaches, including STAMP, that deliberately abstract away from the chain of events leading to an accident [3]. Further work is required to determine whether these alternate approaches offer greater or lesser degrees of consistency when applied by different investigators.

The work of all three analysts in building the Time-Actor diagrams that represent accident events was hindered by a lack of evidence following this accident. The pilot received fatal injuries when the airplane impacted the mountains and could not, therefore, provide direct evidence about the course of the accident. The witness statements from the four surviving passengers were of limited value; it was difficult for them to form a clear view of the events and other factors influencing the pilot's behaviour. The airplane did not have a flight recorder system installed,

As with Analyst A, the focus of the second investigators work also settled on the pilot's intracerebral hemorrhage (ICH) and the subsequent decision to reissue his aviation certificate after medical and neurological evaluations. The results from the evaluation indicated that the accident pilot had fully recovered from the $\mathrm{ICH}$, and that he did not have any neurological deficits. The Federal Aviation Administration indicated that they at the time had issued a total of 19 firstclass airman medical certificates to pilots following ICH. However, Analyst B focussed more directly on the possibility that the accident pilot had experienced a medical condition leading to transient incapacitation or impairment, as a contributing factor to the accident. An autopsy performed on the accident pilot did not identify any direct evidence of such a problem. Two independent neurologists confirmed the lack of evidence that the accident pilot experienced a specific medical event or condition during the accident flight. This contrasts with the first analysis, which placed greater weight on the previous medical history than on the absence of direct medical evidence for transient incapacitation or impairment following the crash. This difference of emphasis is important because it typifies the conflicts that can arise in accident teams where there may often be insufficient or contradictory evidence available in complex investigations.

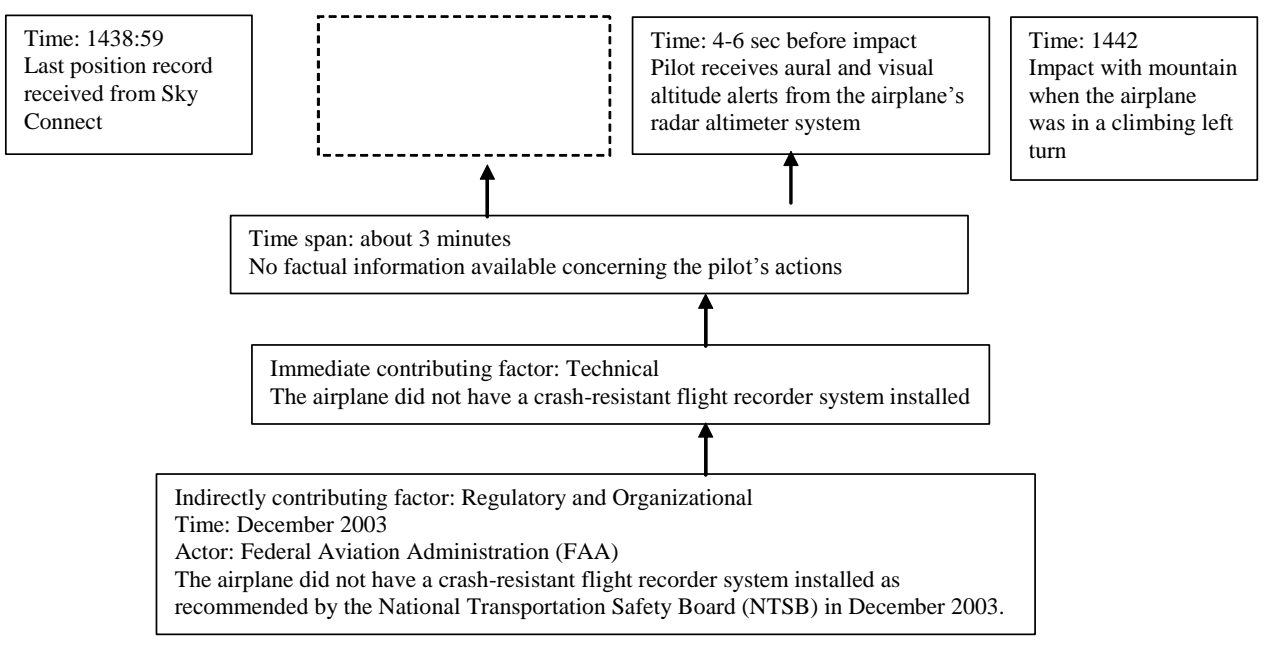

Figure 3: SOL Time-Actor Diagram for Analyst B Illustrating the Lack of Evidence about the Causes of the Accident

Figure 3 illustrates the results from Analyst B's application of the SOL technique. As the factual investigation did not establish any exact evidence to determine what actually happened in the critical minutes before impact, Analyst B regarded the causes as entirely speculative. The lack of observable facts leading up to the accident is represented by an empty event block in the diagram. This is not a standard part of the SOL technique. However, the use of such notational extensions is common in investigatory boards where local adaptations are often encouraged to meet the demands of particular accidents. However, they also illustrate the potential for further inconsistency when others do not exploit the same notational adaptations used by their colleagues.

Analyst B focused on lack of observable facts about events leading to the accident, the first alarm or the first perceived deviation from a warranted course or action. In this case the accident pilot's actions in the 3-minute period between the airplane's last reported position and the time of impact remains unknown. However, this lack of evidence raised further questions for Analyst B. The absence of observable 
facts stems from a host of regulatory and organizational concerns. Valuable information about the accident pilot's last actions could have been provided if a flight recorder system was installed in the airplane.

In 2003 the National Transportation Safety Board (NTSB) issued a safety recommendation asking the Federal Aviation Administration (FAA) to require that such equipment be installed on aircraft like the accident airplane. The FAA, however, did not implement this recommendation. This decision made by the FAA is regarded as the indirectly contributing factor, which impedes organizational learning in order to enhance safety and prevent future accidents. Again, this focus reflects further differences between Analyst A and Analyst B. By expanding the scope of the investigation to question the lack of evidence, this second application of SOL illustrates the benefits to be derived from allowing multiple investigators to apply a similar toolset to the same accident [2]. The individual differences between investigators, identified by Lekberg [5], survive the application of tools and techniques that have been developed to ensure the consistency of causal analysis.

\section{Analyst C}

The third investigator had previously used a number of different analytic techniques for casual factor identification. However, this case study was their first attempt to use SOL. The application of causal analysis techniques can raise a host of problems; many approaches lack detailed technical documentation and training material. A number of documents provide case studies in the application of SOL [2, 6]. It can still be difficult to apply the concepts and ideas to new accidents and incidents. It is for these reasons that Analyst C's initial time-actor diagram was relatively simple, as shown in Figure 4. They justified this sketch using similar arguments to those introduced by the second investigator. They believed that no other pertinent and certain information could be added to the Time-Actor diagram in Figure 4, based on the evidence that was gathered in the aftermath of the accident.

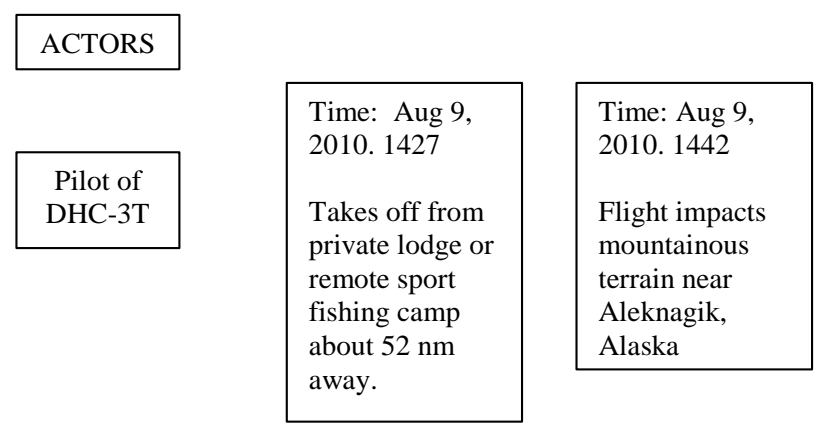

Figure 4: Initial SOL Time-Actor Diagram for Analyst C

The lack of evidence about the immediate causes of the accident led Analyst $\mathrm{C}$ to create a new time-actor diagram focussing on the NTSB's inability to determine why the pilot was temporarily unresponsive. This diagram is shown in
Figure 5. As can be seen, it builds on many of the concerns identified by Analyst B as a result of their findings derived from Figure 3. Figure 5 begins nearly seven years before the crash, when the NTSB first recommended that the FAA require aircraft such as the accident aircraft be equipped with flight recorders and proceed through similar recommendations. The diagram also focuses on the FAA's decision to not implement these recommendations. Much more could be said about the regulatory, organisational and governmental pressures that influence the different decisions and recommendations made in the aftermath of this and similar accidents. Brevity prevents this more sustained presentation. It is only possible to provide an outline of the individual differences between the investigators in a conference paper. However, a journal paper is presently being developed to document the extended results from our comparisons.

\section{Comparisons and Analysis}

Previous sections have presented the results from three different analysts applying SOL to the same incident. The following findings can be identified from our initial study.

Differences in the scope of the analysis - the first investigator focussed on certification of the accident pilot. In contrast, the remaining two analysts argued that there was no direct evidence of the pilot being incapacitated and instead looked at the reasons why there was insufficient evidence about the events leading to the mishap.

Differences in interpreting absent information - the investigators took different approaches to the lack of evidence about the causes of the accident. Analysts B and C took a strict view; they restricted their analysis to those events for which there is direct evidence. It can be argued that to go beyond the available evidence is to risk speculating about the cause of an accident. In contrast, Analyst A argued that the accident provided 'learning opportunities' from apparent weaknesses in the medical re-certification of aircrew even if there was no evidence to suggest that the pilot had been incapacitated in this accident.

Differences in the Use of the SOL Notation - the three analysts worked independently during the study. Partly in consequence, it is possible to see a number of differences in their use of the Time-Actor diagrams. These include the level of detail, for example between Figures 2 and 4 . The analysts also introduced new features to help them model aspects of this accident, including the blank event in Figure 3 by Analyst $\mathrm{B}$ to denote the lack of evidence. As mentioned, these changes increase the flexibility of the SOL approach in order to represent and reason about particular mishaps. However, they can undermine consistency that might otherwise enable wider comparisons between the analyses of different accidents by different investigatory agencies using SOL.

Differences in Confidence and Experience Using SOL -after the study, the three investigators reported different levels of 
confidence in their application of the technique. This can be explained by different levels of expertise in applying the technique and also by the continuing need to support causal analysis techniques with appropriate training and documentation [4].

Differences between Alternate Techniques - this study arguably raised more questions than it answered. In particular, it remains to be seen whether the independent application of different techniques by a groups of investigators working on the same incident would also yield differences similar to those summarised in this section. Other questions relate to international differences between investigators. This is particularly important given that global markets have led to mishaps being repeated in different countries [3]. This paper and the Bielefeld workshops have provided some insights into these topics [1]; however, much remains to be done.

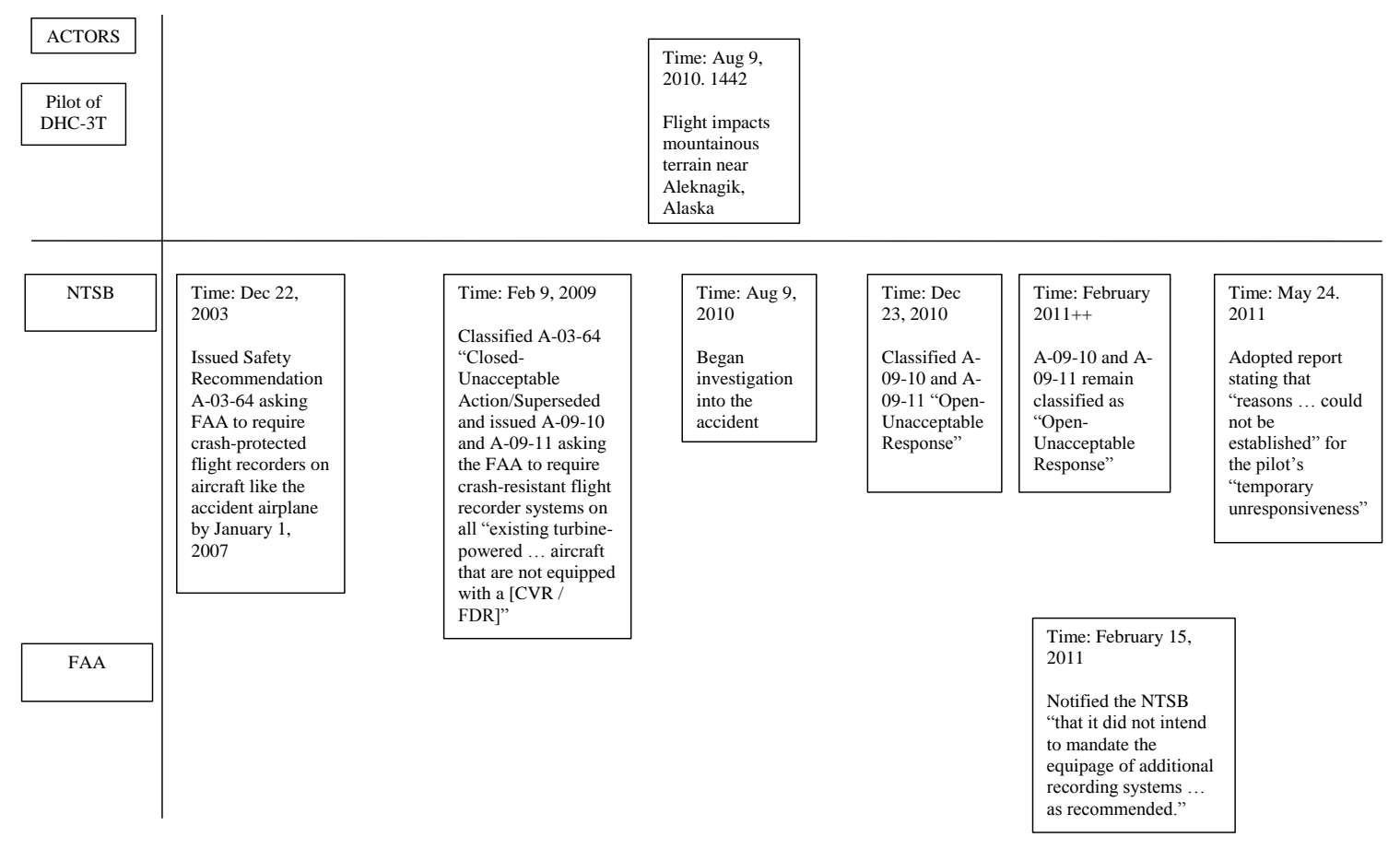

Figure 5: SOL Time-Actor Diagram for Analyst C

\section{Conclusions and Further Work}

Accident reports preserve safety by presenting recommendations that can help avoid any recurrence of past failures. The value of these findings depends upon the causal analysis that explains why an accident occurred. This paper has identified some of the differences that arise when investigators independently apply an analysis technique to the same accident. The results show that different investigators focus on different aspects of an accident; this confirms previous work by Lekburg [5]. Our work also revealed differences in the interpretation of missing evidence, differences in the use of common notations and differences in the level of confidence in the approach. A key finding from this study has been to question the way in which previous studies emphasise the benefits of causal analysis tools in enhancing consistency [3]. Not only can it be shown that individual differences persist in the use of these techniques but it can also be argued that this is a strength; encouraging teams to explore a wide range of lessons from previous accidents.

\section{References}

[1] Braband J, de Stefano E., Kurz S-L, A Comparison of Event-Based Root Cause Analysis Methods. Siemens Transportation Systems, Technical University, Braunschweig, Germany, 2004.

[2] Fahlbruch, B., Wilpert, B., 1997. Event analysis as problem solving process. In: Hale, A., Wilpert, B., Freitag, M. (Eds.), After the Event. Elsevier Science, Oxford, pp. 113-129.

[3] Johnson, C.W., A Handbook of Accident and Incident Reporting, Glasgow University Press, 2003. Available from: http://www.dcs.gla.ac.uk/ johnson/book

[4] Johnson, C.W., Competency Management Systems to Support Accident and Incident Investigators. In C. G. Muniak (ed.), Proc. of the 29th Int Systems Safety Society, Unionville, VA, USA, 2011.

[5] Lekberg, A., 1997. Different Approaches to Accident Investigation: How the Analyst Makes the Difference. In Proceedings of the 15th Int Systems Safety Conference. Sterling, VA.

[6] NTSB, Collision into Mountainous Terrain, GCI Communication Corp. de Havilland DHC-3T, N455A, 
Aleknagik, Alaska, August 9, 2010. Washington DC, Accident Report NTSB/AAR-11/03, PB2011-910403.

[7] Bernhard Wilpert, SOL - Safety through Organizational Learning. A computer assisted Event Analysis Methodology, Research Center System Safety, Berlin University of Technology. 